\title{
JUST EXCELLENCE AND VERY EXCELLENCE IN GRAPHS WITH RESPECT TO STRONG DOMINATION
}

\author{
C. V. R. HARINARAYANAN, C. Y. PONNAPPAN, S. P. SUBBIAH, \\ R. SUNDARESWARAN AND V. SWAMINATHAN \\ $\|$
}

\begin{abstract}
A graph $G$ is said to be excellent with respect to strong domination if each $u \in V(G)$, belongs to some $\gamma_{s}$-set of $G$. $G$ is said to be just excellent with respect to strong domination if each $u \in V(G)$ is contained in a unique $\gamma_{s}$-set of $G$. A graph $G$ which is excellent with respect to strong domination is said to be very excellent with respect to strong domination if there is a $\gamma_{s}$-set $D$ of $G$ such that to each vertex $u \in V-D$, there exists a vertex $v \in D$ such that $(D-\{v\}) \cup\{u\}$ is a $\gamma_{s}$-set of $G$. In this paper we study these two classes of graphs. A strong very excellent graph is said to be rigid very excellent with respect to strong domination if the following condition is satisfied. Let $D$ be a very excellent $\gamma_{s}$-set of $G$. To each $u \notin D$, let $E(u, D)=\left\{v \in D:(D-\{v\}) \cup\{u\}\right.$ is a $\gamma_{s}$-set of $\left.G\right\}$. If $|E(u, D)|=1$ for all $u \notin D$ then $D$ is said to be a rigid very excellent $\gamma_{s}$-set of $G$. If $G$ has at least one rigid very excellent $\gamma_{s}$-set of $G$ then $G$ is said to be a rigid very excellent graph with respect to strong domination (or) a strong rigid very excellent graph. Some results regarding strong very excellent graphs are obtained.
\end{abstract}

\section{Introduction}

Prof. N. Sridharan and M. Yamuna have introduced the concepts of just excellence and very excellence in graphs. A graph $G$ is said to be excellent if given any vertex $u$, there is a $\gamma$-set of $G$ containing $u$. A graph $G$ is said to be just excellent if for each vertex $u \in V$, there is a unique $\gamma$-set of $G$ containing $u$. A graph $G$ is very excellent if $G$ is excellent and if there is a $\gamma$-set $S$ of $G$ such that to each vertex $u \in V-S$, there exists a vertex $v \in S$ such that $(S-\{v\}) \cup\{u\}$ is a $\gamma$-set of $G$. A $\gamma$-set $S$ of $G$ satisfying this property is called a very excellent $\gamma$-set of $G$.

Prof. E. Sampathkumar and Pushpalatha have introduced the concept of Strong (weak) domination. A subset $D$ of $V(G)$ is called a strong dominating set if for every vertex $v \in V-D$, there exists $u \in D$ such that $u v \in E(G)$ and $\operatorname{deg} u \geq \operatorname{deg} v$. A strong dominating set of minimum cardinality is called a minimum strong dominating set and its cardinality is called the strong domination number. The strong domination number is denoted by $\gamma_{s}$ and a minimum strong dominating set is called $a \gamma_{s}$-set.

A subset $D$ of $V(G)$ is called a weak dominating set of $G$ if for every vertex $v \in V-D$, there exists $u \in D$ such that $u v \in E(G)$ and $\operatorname{deg} u \leq \operatorname{deg} v$. A weak dominating set of minimum cardinality is called a minimum weak dominating set and its cardinality is

Received December 27, 2005; revised September 20, 2006. 
called the weak domination number. The weak domination number is denoted by $\gamma_{w}$ and a minimum weak dominating set is called a $\gamma_{w}$-set.

Definition 1. A graph $G$ is said to be strong excellent $\gamma_{s}$-excellent, if for a given vertex $u$ of $G$ there exists a $\gamma_{s}$-set of $G$ containing $u$.

Definition 2. A graph $G$ is said to be strong just excellent (or) shortly $\gamma_{s}$-just excellent if for every $u \in V$, there is a unique $\gamma_{s}$-set containing $u$.

Definition 3. A graph $G$ is said to be strong very excellent (or) shortly $\gamma_{s}$-very excellent if there is a $\gamma_{s}$-set $S$ of $G$ such that to each vertex $u \notin S$, there exists $v \in S$ with $(S-\{v\}) \cup\{u\}$ a $\gamma_{s}$-set of $G$. A $\gamma_{s}$-set $S$ of $G$ satisfying this property is called $a$ very excellent $\gamma_{s}$-set of $G$.

Definition 4. A graph $G$ is said to be strong rigid very excellet (or) shortly $\gamma_{s}$-rigid very excellent, if $G$ is strong very excellent and for any very excellent $\gamma_{s}$-set $D$ of $G$ and for any $u \notin D$ there exists a unique $v \in D$ such that $(D-\{v\}) \cup\{u\}$ is a $\gamma_{s}$-set.

Definition 5. Let $u$ and $v$ belong to $V(G)$. Then $\operatorname{deg}_{s}(u)=\left|N_{s}(u)\right|$ where $N_{s}(u)=$ $\{v \in V: u v \in E(G), \operatorname{deg} v \geq \operatorname{deg} u\}$. Similarly $N_{w}(u)$ is defined as $N_{w}(u)=\{v \in V:$ $u v \in E(G), \operatorname{deg} v \leq \operatorname{deg} u\} . \quad d_{w}(u)$ is defined as $d_{w}(u)=\left|N_{w}(u)\right| . \quad u$ is said to be a strong isolate if $N_{s}(u)=\phi$. Similarly a weak isolate can be defined.

Definition 6. $\delta_{s}(G)=\min _{u \in V}\left(d_{s}(u)\right), \Delta_{s}(G)=\max _{u \in V}\left(d_{s}(u)\right), \delta_{w}(G)=\min _{u \in V}\left(d_{w}(u)\right)$ and $\Delta_{w}(G)=\max _{u \in V}\left(d_{w}(u)\right)$.

Definition 7. If $D$ is a $\gamma_{s}$-set of $G$, then $P N_{w}[u, D]=\{v \in V(G): v$ is strongly dominated by $u$ and $v$ is not strong dominated by $D-\{u\}\}=N_{w}[u]-N_{w}[D-\{u\}]$. $P N_{w}(u, D)$ is defined as $N_{w}(u)-N_{w}[D-\{u\}]$. Note that $u \in P N_{w}[u, D]$ and $u \notin$ $P N_{w}(u, D)$.

Example 1. Any $\gamma$-excellent regular graph is $\gamma_{s}$-excellent.

Example 2. Any double star $K_{r, r}$ is $\gamma_{s}$-rigid excellent but not $\gamma$-rigid excellent.

Example 3. $K_{n}$ is $\gamma_{s}$-very excellent.

xample $4 . G=$

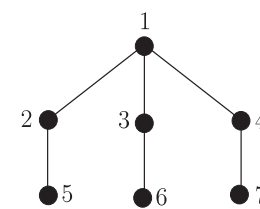

is $\gamma_{s}$-very excellent, since $\{1,2,3,4\}$ is a $\gamma_{s}$-set. $\{1,3,4,5\},\{1,2,4,6\},\{1,2,3,7\}$ are $\gamma_{s}$-sets. $G$ is not $\gamma$-very excellent.

Example 5. $G={ }_{3}^{2}$ This is $\gamma$-just excellent but not $\gamma_{s}$-just excellent. 
Example 6. $G=$

\section{Just Excellent Graphs}

Observation 1. If $G$ is $\gamma_{s}$-just excellent and $G \neq K_{n}$ then $N_{w}[u] \neq N_{w}[v]$ for any $u, v \in V(G)$, where $\left\{N_{w}[u]=\{\{u\} \cup\{v \in V: u v \in E(G) ; d(u) \geq d(v)\}\}\right.$.

Proof. Since $G$ is $\gamma_{s}$-just excellent, there exists a unique $\gamma_{s}$-set say $D$ containing $u$. Suppose there exists a $v \in V(G)$ such that $N_{w}[u]=N_{w}[v]$. Then $(S-\{u\}) \cup\{u\}$ is a $\gamma_{s}$-set. Since $G \neq K_{n}$ and since any non-regular graph with a vertex of degree $(n-1)$ is not $\gamma_{s}$-rigid excellent, $|S|>1$. Therefore, every vertex of $S-\{u\}$ lies in at least two $\gamma_{s}$-excellent sets namely $S$ and $(S-\{u\}) \cup\{v\}$ contradicting the $\gamma_{s}$-rigid excellence of $G$. Hence the observation.

Observation 2. If $G$ is $\gamma_{s}$-excellent then $\delta_{s}(u) \geq \frac{n}{\gamma_{s}(G)}-1$.

Proof. Let us assume that $V=S_{1} \cup S_{2} \cup \cdots \cup S_{m}$ where each $S_{i}$ is a $\gamma_{s}$-set. Let $m \geq 2$. Let $u \in S_{j}$. Since each $S_{i}$ is a $\gamma_{s}$-set, $u$ is strongly dominated by some point $v \in S_{i}, i \neq j$. Hence $\delta_{s}(u) \geq m-1=\frac{n}{\gamma_{S}(G)}-1$. If $m=1$ the $V$ is a $\gamma_{s}$-set. Hence $G$ is totally disconnected. Therefore, $\delta_{s}(u)=0=\frac{n}{n}-1=\frac{n}{\gamma_{s}(G)}-1$.

Observation 3. If $G \neq K_{2}$ and $G \neq \overline{K_{n}}$ and if $G$ is $\gamma_{s}$-just excellent then $\delta_{s}(u) \geq 2$ (In particular any tree $\neq K_{2}$ is not $\gamma_{s}$-just excellent).

Proof. Let $G \neq K_{2}, G \neq \overline{K_{n}}$. Let $\delta_{s}(u)=1$ for some $u \in V(G)$. Let $N_{s}(u)=\{v\}$. Since $G$ is just excellent there exists a $\gamma_{s}$-set $D$ of $G$, containing $u$. If $v \in D$, then there are two $\gamma_{s}$-sets containing $v$, since $D$ and $(D-\{u\}) \cup\{v\}$ are two $\gamma_{s}$-sets containing $v$. Therefore, $v \notin D$, since $G \neq K_{2},|D| \geq 2$. Therefore, $(D-\{u\}) \cup\{v\}$ is a $\gamma_{s}$-set or $G$ and hence every element of $D-\{u\}$ is contained in at least two $\gamma_{s}$-sets, a contradiction.

Lemma 1. Every $\gamma_{s}$-just excellent graph $G \neq \overline{K_{n}}$ is connected.

Proof. If $G$ is not connected, by hypothesis, one of the connected components say $G_{1}$ of $G$ contains more than one vertex. Since $G$ is $\gamma_{s}$-just excellent, $G_{1}$ is $\gamma_{s}$-just excellent and $G_{1}$ is connected, $\gamma_{s}\left(G_{1}\right)<\left|V\left(G_{1}\right)\right|$. Since $G_{1}$ is $\gamma_{s}$-just excellent, $G_{1}$ has at least two $\gamma_{s}$-sets. Let $S_{1}, S_{2}$ be two $\gamma_{s}$-sets of $G_{1}$. Let $D$ be a $\gamma_{s}$-set of $G-G_{1}$. Then both $D \cup S_{1}$ and $D \cup S_{2}$ are $\gamma_{s}$-set of $G$ containing $D$, a contradiction to the fact that $G$ is $\gamma_{s}$-just excellent. Hence $G$ is connected.

Lemma 2. If $G$ is strong just excellent and $G \neq \overline{K_{n}}$, then $G$ has no strong isolates. 
Proof. Since $G$ is strong just excellent and $G \neq \overline{K_{n}}$, by the above lemma, $G$ is connected. Then $\gamma_{s}(G)<|V(G)|$. Therefore $G$ has at least two $\gamma_{s}$-sets. If $G$ has a strong isolate, then this belongs to every $\gamma_{s}$-set, a contradiction. Hence $G$ has no strong isolates.

Definition 8. Let $D$ be a subset of $V$. Then $\langle D\rangle$, called the induced subgraph of $G$, is defined as the subgraph with vertex set $D$ and two vertices in this subgraph are adjacent if they are adjacent in $G$.

Lemma 3. If $G \neq K_{n}$ and $G$ is $\gamma_{s}$-just excellent then $\left|P N_{w}(u, D)\right| \geq 2$ for all $u \in D$, where $D$ is a $\gamma_{s}$-set of $G$, and $u$ is not a strong isolate of $\langle D\rangle$.

Proof. Let $D$ be a $\gamma_{s}$-set of $G$. If $P N_{w}(u, D)=\phi$, then $(D-\{u\}) \cup\{w\}$ is also a $\gamma_{s}$-set of $G$, for any $w$ in $N_{s}(u)$. (Note that $N_{s}(u) \neq \phi$ as $G$ has no strong isolates). If $D=\{u\}$ then, $G=K_{n}$ a contradiction. Therefore, $D-\{u\}$ contains a point and hence every point in $D-\{u\}$ is contained in at least two $\gamma_{s}$-sets, namely $D$ and $(D-\{u\}) \cup\{w\}$, a contradiction since $G$ is strong just excellent. Suppose $\left|P N_{w}(u, D)\right|=$ 1. Let $P N_{w}(u, D)=\{w\}$.

Then $(D-\{u\}) \cup\{w\}$ is a $\gamma_{s}$-set (since $u$ is not a strong isolate of $\langle D\rangle$ ). Noting that $D$ has at least two points we get that every vertex in $(D-\{u\})$ is in at least two $\gamma_{s}$-sets, namely $D$ and $(D-\{u\}) \cup\{w\}$, a contradiction. Hence the theorem.

Remark 1. If $G$ is $\gamma_{s}$-just excellent and if $S$ is a $\gamma_{s}$-set of $G$, then a vertex in $V-S$ may be strong dominated by more than one vertex of $S$. For example, in Example 6, the vertex 2 is strong dominated by two vertices of the $\gamma_{s}$-set $\{1,3,6\}$.

Theorem 1. Let $G \neq \overline{K_{n}}$ be just excellent. Let $\gamma_{s}(G)=k$. Then $\Delta_{w}(G) \leq n-k$.

Proof. Let $u \in V(G)$. Let $S$ be a $\gamma_{s}$ set of $G$ which contains $u$. $\left|P N_{w}(V-S)\right| \geq 1$ for all $v \in S$. Therefore, $u$ is not strong adjacent to any point in $\bigcup_{v \neq u, v \in S} P N_{w}(v, S)$. Therefore, $d_{w}(u) \leq(n-1)-(k-1)=n-k$. Therefore, $\Delta_{w}(G) \leq n-k$.

Definition 9. The strong domatic number of $G$, denoted by $d_{s}(G)$ is defined as the maximum cardinality of partition of $V$ into strong dominating sets of $G$. Note that since $V$ is a strong dominating set $d_{s}(G) \geq 1$.

Lemma 4. The graph $G$ is just excellent if and only if all of the following conditions hold.

1. $\gamma_{s}(G)$ divides $n$.

2. G has exactly $\frac{n}{\gamma_{s}(G)}$ distinct $\gamma_{s}$-sets.

3. $d_{s}(G)=\frac{n}{\gamma_{s}(G)}$.

Proof. Let $G$ be just excellent. Let $S_{1}, S_{2}, S_{3}, \ldots, S_{m}$ be the collection of distinct $\gamma_{s}$-sets of $G$. Then $S_{1}, S_{2}, S_{3}, \ldots, S_{m}$ is a partition of $V$ into $m \gamma_{s}$-sets. Therefore 
$m \gamma_{s}(G)=n$. Therefore (1) and (2) follows. Since $S_{1}, S_{2}, S_{3}, \ldots, S_{m}$ provide a domatic partition with $m=\frac{n}{\gamma_{s}(G)}$ we get that $d_{s}(G)=\frac{n}{\gamma_{s}(G)}$.

Conversely assume that $G$ satisfies conditions 1-3. Then $m \gamma_{s}(G)=n$. Since $d_{s}(G)=$ $\frac{n}{\gamma_{s}(G)}=m$, there exists a decomposition of $V(G)$ into $m$ strong dominating sets of $G$, say $S_{1}, S_{2}, S_{3}, \ldots, S_{m}$. Then $\left|S_{i}\right| \geq \gamma_{s}$. Therefore $n=\sum_{i=1}^{m}\left|S_{i}\right| \geq m \gamma_{s}$. Therefore $m \gamma_{s}(G)=\sum_{m}^{i=1}\left|S_{i}\right| \geq m \gamma_{s}$. Therefore each $S_{i}$ is a $\gamma_{s}$-set. By hypothesis $G$ has exactly $\frac{n}{\gamma_{s}(G)}$ distinct $\gamma_{s}$-sets. That is $G$ has exactly $m$ distinct $\gamma_{s}$-sets. Therefore, $S_{1}, S_{2}, S_{3}, \ldots, S_{m}$ are precisely $m$ distinct $\gamma_{s}$-sets. Also each vertex belongs to exactly one $S_{i}$ (since $\left\{S_{1}, S_{2}, S_{3}, \ldots, S_{m}\right\}$ is a partition of $V$ ). Therefore, $G$ is $\gamma_{s}$-just excellent.

Theorem 2. Let $u \in V$. Let $D$ be the unique $\gamma_{s}$-set containing $u$. Let $t$ be the number of strong isloates of $\langle D\rangle$.

Then $d_{w}(u) \leq \begin{cases}n-2 \gamma_{s}+2 t-1 & \text { if } u \text { is not a strong isolate of } D \\ n-2 \gamma_{s}+2 t-3 & \text { if } u \text { is a strong isolate of } D\end{cases}$

Proof. For any non-strong isolate $v$ of $D,\left|P N_{w}(v, D)\right| \geq 2$. Also, if $v \in D$ and if $x \in P N_{w}(v, D)$ then $u$ does not strong dominate $x$.

Therefore $d_{w}(u) \leq \begin{cases}(n-1)-2\left(\gamma_{s}-1-t\right)+t & \text { if } u \text { is not a strong isolate, } \\ (n-1)-2\left[\gamma_{s}-1-(t-1)\right]+(t-1) & \text { if } u \text { is a strong isolate. }\end{cases}$

Therefore $d_{w}(u) \leq \begin{cases}n-2 \gamma_{s}+3 t+1 & \text { if } u \text { is not a strong isolate, } \\ n-2 \gamma_{s}+3 t-2 & \text { if } u \text { is a strong isolate. }\end{cases}$

Corollary 1. If $D$ has no strong isolates then $d_{w}(u) \leq n-2 \gamma_{s}+1$.

Theorem 3. Let $G \neq K_{n}$ be just excellent. Then $\gamma_{s}(G) \leq \frac{n}{3}$.

Proof. Suppose $d_{s}(G)=2$. Then $V=S_{1} \cup S_{2}$ where $S_{1}$ and $S_{2}$ are distince $\gamma_{s}$-sets of $G$. For any $u \in S_{1}, P N_{w}\left(u, S_{1}\right) \subseteq S_{2}$. Suppose for some $u \in S_{1},\left|P N_{w}\left(u, S_{1}\right)\right| \geq 2$. Then $\left|S_{2}\right| \geq\left|S_{1}\right|+1$. But $2 \gamma_{s}=\left|S_{1}\right|+\left|S_{2}\right| \geq 2\left|S_{1}\right|+1 \geq 2 \gamma_{s}+1$, a contradiction. Therefore, every point of $S_{1}$ is a strong isolate of $S_{1}$. Similarly every point of $S_{2}$ is a strong isolate of $S_{2}$. Suppose $\left|P N_{w}\left(u, S_{1}\right)\right| \geq 2$ for some point $u \in S_{1}$, by the above argument we get that $2 \gamma_{s} \geq \gamma_{s}+1$, a contradiction. Therefore, $\left|P N_{w}\left(u, S_{1}\right)\right|=1$ for $u \in S_{1}$. Similarly this result is true for $S_{2}$ also.

Let $S_{1}=\left\{u_{1}, u_{2}, u_{3}, \ldots, u_{k}\right\}, S_{2}=\left\{v_{1}, v_{2}, v_{3}, \ldots, v_{k}\right\}$. Without loss of generality let $\left\{v_{i}\right\}=P N_{w}\left(u_{i}, S_{1}\right)$. Then $d\left(u_{i}\right)>d\left(v_{i}\right)$. If $d\left(u_{i}\right)=d\left(v_{i}\right)$ then $\left(S_{1}-\left\{u_{i}\right\}\right) \cup\left\{v_{i}\right\}$ is a $\gamma_{s^{-}}$ set and so, every point of $\left(S_{1}-\left\{u_{i}\right\}\right)$ lies in two $\gamma_{s}$-sets namely $S_{1}$ and $\left(S_{1}-\left\{u_{i}\right\}\right) \cup\left\{v_{i}\right\}$, a contradiction.

Suppose $u_{i}$ and $u_{j}$ are adjacent, as $u_{i}$ and $u_{j}$ are strong isolates, $d\left(u_{i}\right)>d\left(u_{j}\right)$ and $d\left(u_{j}\right)>d\left(u_{i}\right)$, a contradiction. Therefore, $u_{i}$ and $u_{j}$ are not adjacent. That is $\left\langle S_{1}\right\rangle$ is totally disconnected. The same is true for $S_{2}$ also. Let $u_{1}, u_{2}, \ldots, u_{k}$ be such that $d\left(u_{1}\right) \leq d\left(u_{2}\right) \leq \cdots \leq d\left(u_{k}\right)$. We have $d\left(u_{1}\right)>d\left(v_{1}\right)$. Also $d\left(v_{1}\right)>d\left(u_{s}\right)$ for some $s>1$ (where $\left\{u_{s}\right\}=P H_{w}\left(v_{1}, S_{2}\right)$ ). Therefore, $d\left(u_{1}\right)<d\left(u_{s}\right)<d\left(v_{1}\right)<d\left(u_{1}\right)$, a contradiction. Therefore $d_{s}(G) \geq 3$. Since $n=\gamma_{s}(G) d_{s}(G)$, we get that $\gamma_{s}(G)=\frac{n}{d_{s}(G)} \leq \frac{n}{3}$. 
Remark 2. For $C_{3 n}, \gamma_{s}\left(C_{3 n}\right)=n$ and $C_{3 n}$ is $\gamma_{s}$-just excellent.

Definition 10. Let $u \in V(G)$. A subset $S$ of minimum cardinality such that $S$ strong dominates $G-\{u\}$ is called $a \gamma_{s}^{u}(G, u)$ set of $G$.

Definition 11. $u \in V$ is said to be a $\gamma_{s}$ level vertex of $G$, if $\gamma_{s}^{u}(G, u)=\gamma_{s}(G)$. $u$ is said to be a $\gamma_{s}$-non-level vertex of $G$, if $\gamma_{s}^{u}(G, u)=\gamma_{s}(G)-1$.

Example 7. $G$ :

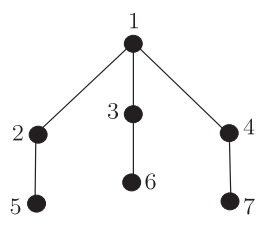

Example 8. $G$ :

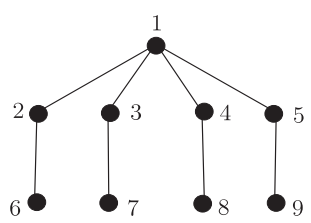

$G-2:$

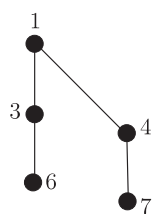

$G-6:$

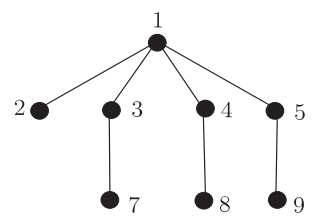

In Example 7, $\{2,3,4\}$ and $\{3,4,5\}$ are subsets of $V$ of minimum cardinality which dominate $G-\{2\}$. Therefore $\gamma_{s}^{2}(G, 2)=3$. 2 is a $\gamma_{s}$-non level vertex of $G$. In Example $8 \gamma_{s}(G)=\gamma_{s}^{8}(G, 8)=5$. Therefore 8 is a $\gamma_{s}$-level vertex.

Theorem 4. Let $G$ be a $\gamma_{s}$-just excellent graph, $G \neq \overline{K_{n}}$. Then every vertex $u$ is a $\gamma_{s}$-level vertex and $\gamma_{s}(G-\{u\})=\gamma_{s}(G)$.

Proof. If $G=K_{n}$, the theorem is obviously true. Let $G \neq K_{n}$ and $G \neq \overline{K_{n}}$. Let $u$ be a vertex in $G$. Since $G$ is $\gamma_{s}$-just excellent, there exists a $\gamma_{s}$-set $S$ of $G$ not containing $u$. Clearly $S$ strong dominates $G-\{u\}$. Therefore, $\gamma_{s}(G-\{u\}) \leq|S| \leq \gamma_{s}(G)$. Suppose $\gamma_{s}(G-\{u\})<\gamma_{s}(G)$. Let $T$ be a $\gamma_{s}$-set of $G-\{u\}$. Then $T \cup\{v\}$ is a $\gamma_{s^{-}}$set for $G$, for every $v \in N_{s}[u]$. $N_{s}[u]$ contains at least two points, since $u$ is not a strong isolate. Therefore, there exists a point in $N_{s}[u]$ different from $u$ which strong dominates $u$. Let $v \in N_{s}(u)$. Then $T \cup\{v\}$ and $T \cup\{u\}$ are $\gamma_{s}$-sets containing $T$. Therefore, every element of $T$ is contained in at least two $r_{s}$-sets of $G$, a contradiction to $\gamma_{s}$-just excellence of $G$. Therefore, $\gamma_{s}(G-\{u\})=\gamma_{s}(G)$.

Suppose $\gamma_{s}^{u}(G, u)<\gamma_{s}(G)$. Let $S \subseteq V$ be a $\gamma_{s}^{u}(G, u)$-set of $G$. If $u \in S$, then $S$ is $\gamma_{s}$-set of $G$, a contradiction. Therefore, $u \notin S$. Therefore $S$ is a strong dominating set for $G-u$. Therefore, $\gamma_{s}(G-\{u\}) \leq|S|<\gamma_{s}(G)$, a contradiction. Since $\gamma_{s}(G-\{u\})=\gamma_{s}(G)$, $\gamma_{s}^{u}(G,\{u\})=\gamma_{s}(G)$. Hence the theorem.

\section{Strong Very Just Excellent Graphs}

We recall the definition of strong very excellent (or) $\gamma_{s}$-very excellent graphs. 
A strong excellent graph $G$ is said to be strong very excellent if there is a $\gamma_{s}$-set $S$ of $G$ such that to each vertex $u \in V-S$, there exists a vertex $v$ in $S$ such that $(S-\{v\}) \cup\{u\}$ is a $\gamma_{s}$-set of $G$. A $\gamma_{s}$-set of $G$ satisfying the above property is called very just excellent $\gamma_{s}$ set of $G$.
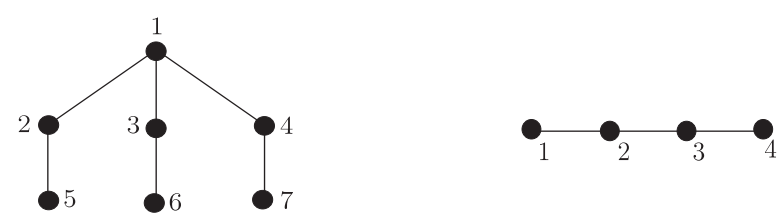

In first example, the graph is strong very just excellent and $\{1,2,3,4\}$ is a very just excellent $\gamma_{s}$-set. In second example $\{1,3\},\{2,3\},\{2,4\}$ is very excellent and $\{2,3\}$ is very just excellent $\gamma_{s}$-set.

Theorem 5. $P_{n}$ is $\gamma_{s}$-very excellent if and only if $n=2$ or $n=4$.

Proof. It has already been proved in [2] that $P_{n}$ is $\gamma_{s}$-excellent if and only if $n=$ 2 or $n \equiv 1(\bmod 3) . \quad P_{2}, P_{4}$ are obviously $\gamma_{s}$-very excellent. Consider a path $P_{n}$ : $v_{1}, v_{2}, v_{3}, \ldots, v_{n}$ where $n=3 k+1, k \geq 2$. Let $S$ be any $\gamma_{s}$-set for $P_{n}$. Then at least $\gamma_{s}-2$ vertices are isolated in $\langle S\rangle$. To each $u \in S$, let $P N_{w}(u)=\left\{v \in V\left(P_{n}\right): N_{s}(v) \cap S=\{u\}\right\}$. Suppose $\left|P N_{w}(u)\right|=2$ for some $u \in S$. Let $v_{i}$ be the point in $S$ such that $\left|P N_{w}\left(v_{i}\right)\right|=2$, $2 \leq i \leq 3 k$ (Note that $d(u) \leq 2$ for all $\left.u \in V\left(P_{n}\right)\right)$.

\section{Subcase(1):}

Suppose $i=2$. Since $v_{1}$ is strongly dominated only by $v_{2}$ in $S, S-\left\{v_{2}\right\} \cup\left\{v_{j}\right\}, j \neq 1$ is not a strong dominating set. Also $\left(S-\left\{v_{2}\right\}\right) \cup\left\{v_{1}\right\}$ is also not a strong dominating set. (For, since $v_{1}, v_{3} \in P N_{w}\left(v_{2}\right)$. $v_{3}$ is a weak private neighbour of $v_{2}$. Since $v_{2}$ is dropped the newly introduced point $v_{1}$ must dominate $v_{3}$ strongly which is not true since $v_{1}$ is not even adjacent to $\left.v_{3}\right)$. This contradicts the fact that $S$ is a strong very excellent $\gamma_{s}$-set. By similar reasoning would prove for $i \neq 3 k$.

\section{Subcase(2):}

Let $2<i<3 k$. It is enough, if we prove for $2<i<\frac{3 k+1}{2}$ (a similar reasoning would prove for $\left.3 k>1>\frac{3 k+1}{2}\right)$. As $\left|P N_{w}\left(v_{i}\right)\right|=2, v_{i-1}, v_{i+1} \in P N_{w}\left(v_{i}\right)$. $v_{i+2} \notin S$ (for if $v_{i+2} \in S$, then $v_{i+1}$ is not in the weak private neighbour of $v_{i+2}$, a contradiction.) Clearly $v_{i+3} \in S$. If $v_{i+3}$ is an isolate in $\langle S\rangle$, then $\left(S-\left\{v_{i+3}\right\}\right) \cup\left\{v_{i+1}\right\}$ does not strong dominate $v_{i+3}$. Then for inclusion of $v_{i+1}$ in $S$, there exists no point in $S$ whose deletion will result in a $\gamma_{s}$-set. Therefore, $v_{i+3}$ is not an isolate in $\langle S\rangle$. Therefore, $v_{i+4} \in S$. Since $n=3 k+1, i+3$ and $i+4$ cannot be the last two points of the path. Therefore, $i+4 \leq n-1$ and $v_{i+5} \in P N_{w}\left(v_{i+4}\right)$. Let $Q_{1}$ denote $v_{1}, v_{2}, \ldots, v_{i-2}$ path and $Q_{2}$ denote $v_{i+6}, v_{i+7}, \ldots, v_{n}$. (Any one of the paths $Q_{1}$ or $Q_{2}$ may be empty). Then 
$\left(S-\left\{v_{i}, v_{i+3}, v_{i+4}\right\}\right)$ dominates the vertices of $Q_{1}$ and $Q_{2}$. As $n \geq 10, Q_{1} \neq \phi$ or $Q_{2} \neq \phi$. $\left|Q_{1} \cup Q_{2}\right|=n-7=3 k+1-7=3 k-6 \equiv 0(\bmod 3)$.

$\left|S-\left\{v_{i}, v_{i+3}, v_{i+4}\right\}\right|=k-2$. And so no vertex in $Q_{1}$ is adjacent to any vertex in $Q_{2},\left\{v_{i}, v_{i+3}, v_{i+4}\right\}$ does not dominate any vertex in $Q_{1} \cup Q_{2}$. The set $S \cap Q_{1}$ strong dominates $Q_{1}$ and $S \cap Q_{2}$ strong dominates $Q_{2}$. So

$$
\begin{aligned}
& \left|S \cap Q_{1}\right|=\left\lceil\frac{\left|Q_{1}\right|}{3}\right\rceil ; \quad\left|S \cap Q_{2}\right|=\left\lceil\frac{\left|Q_{2}\right|}{3}\right\rceil . \\
& \left|S \cap\left(Q_{1} \cup Q_{2}\right)\right|=k-2=\frac{3 k+1-7}{3}=\left|\frac{Q_{1} \cup Q_{2}}{3}\right| .
\end{aligned}
$$

We have $\left|S \cap Q_{1}\right|+\left|S \cap Q_{2}\right|=\left|S \cap\left\{\left(Q_{1} \cup Q_{2}\right)\right\}\right|=\frac{\left|Q_{1} \cup Q_{2}\right|}{3}$. That is, $\left|S \cap Q_{1}\right|+\left|S \cap Q_{2}\right|=$ $\frac{\left|Q_{1} \cup Q_{2}\right|}{3}$. That is, $\left\lceil\frac{\left|Q_{1}\right|}{3}\right\rceil+\left\lceil\frac{\left|Q_{2}\right|}{3}\right\rceil=\frac{\left|Q_{1} \cup Q_{2}\right|}{3}$. Suppose $\left|Q_{1}\right|$ and $\left|Q_{2}\right|$ are not both divisible by 3. Let $\left|Q_{1}\right|=3 l+1$ or $3 l+2$. Let $\left|Q_{2}\right|=3 m+2$ or $3 m+1$ (note that since $\left|Q_{1}\right|+\left|Q_{2}\right|$ is divisible by $3,\left|Q_{1}\right|=3 l+1$ and $\left|Q_{2}\right|=3 m+2$ or $\left|Q_{1}\right|=3 l+2$ and $\left.\left|Q_{2}\right|=3 m+1\right)$. Therefore, $\left\lceil\frac{\left|Q_{1}\right|}{3}\right\rceil+\left\lceil\frac{\left|Q_{2}\right|}{3}\right\rceil=l+1+m+1=l+m+2 . \frac{\left|Q_{1} \cup Q_{2}\right|}{3}=\frac{3 l+1+3 m+2}{3}=l+m+1$, a contradiction.

Suppose $\left|Q_{1}\right|$ is not divisible by 3 and $\left|Q_{2}\right|$ is divisible by 3 . Let $\left|Q_{1}\right|=3 l+1$ or $3 l+2$ and $\left|Q_{2}\right|=3 m .\left\lceil\frac{\left|Q_{1}\right|}{3}\right\rceil+\left\lceil\frac{\left|Q_{2}\right|}{3}\right\rceil=l+1+m+1 . \frac{\left|Q_{1} \cup Q_{2}\right|}{3}=\frac{3 l+1+3 m}{3}$ or $\frac{3 l+3 m+2}{3}$ is not an integer, a contradiction. Similarly $\left|Q_{1}\right|$ is divisible by 3 and $\left|Q_{2}\right|$ is not divisible by 3 is also not true. Therefore, $\left|Q_{1}\right|$ and $\left|Q_{2}\right|$ are divisible by 3. If $Q_{1} \neq \phi$ then $v_{2} \in S$ and $v_{1}, v_{3} \in P N_{w}\left(v_{2}\right)$. Then $(S-\{w\}) \cup\left\{v_{1}\right\}$ is not a strong dominating set for any $w \in S$. If $Q_{2} \neq \phi$, then as $\left|Q_{2}\right|$ is divisible by 3 , we get that $i+4$ or $i+10$ or $\cdots$ or $i+3 t+1$, $(t \geq 2)$ will be the last but one point of the path $P_{n}$. Therefore, $i+3 t+2$ belongs to $S$. That is, $v_{n-1}$ belongs to $S$ and $v_{n-2}, v_{n} \in P N_{w}\left(v_{n-1}\right)$.

In the case for the inclusion of $v_{n} \in S$, there exists no point in $S$ whose deletion will result in a $\gamma_{s}$-set, a contradiction. Therefore, if $S$ is a strong very excellent set, then

$$
\left|P N_{w}(u)\right| \leq 1
$$

for every $u \in S$. Let $n \geq 13$. Let $P^{\prime}$ be the $v_{1}-v_{10}$ path and $P^{\prime \prime}$ be the $v_{11}-v_{n}$ path. If $S \cap P^{\prime}$ does not strong dominate any of the vertices of $P^{\prime \prime}$, then $S \cap P^{\prime \prime}$ is a $\gamma_{s}$-set for $P^{\prime \prime}$. As $\left|p^{\prime \prime}\right| \equiv 0(\bmod 3)$ and $\left\{v_{j}: j=3 t, 4 \leq t \leq k\right\}$ is the unique $\gamma_{s}$ set for $P^{\prime \prime}$. It follows that $v_{n-1} \in S$ and therefore, $\left|P N_{w}\left(v_{n-1}\right)\right|=2$, a contradiction to (1). If $S \cap P^{\prime}$ dominates a vertex of $P^{\prime \prime}$, then $v_{10} \in S$. Hence $\left|S \cap P^{\prime}\right|=4$. If $v_{11} \in P N_{w}\left(v_{10}\right)$, then $S \cap P^{\prime}$ is a $\gamma_{s}$-set for $v_{1}-v_{11}$ path. Then $P N_{w}(u)=2$ for at least two points in $S \cap P^{\prime \prime}$, a contradiction to (1). If $v_{11} \notin P N_{w}\left(v_{10}\right)$, then $v_{12} \in S$ and $S \cap P^{\prime \prime}$. Therefore, $S$ contains $v_{n-1}$ and $\left|P N_{w}\left(v_{n-1}\right)\right|=2$, a contradiction to (1).

If $n=10$, then the $\gamma_{s}$-sets are $S_{1}=\left\{v_{2}, v_{5}, v_{8}, v_{10}\right\}, S_{2}=\left\{v_{2}, v_{5}, v_{8}, v_{9}\right\}, S_{3}=$ $\left\{v_{1}, v_{3}, v_{6}, v_{9}\right\}, S_{4}=\left\{v_{2}, v_{5}, v_{7}, v_{9}\right\}, S_{5}=\left\{v_{2}, v_{4}, v_{7}, v_{9}\right\}$. In $S_{1}$ and $S_{2},\left|P N_{w}\left(v_{5}\right)\right|=2$. In $S_{3},\left|P N_{w}\left(v_{3}\right)\right|=\left|P N_{w}\left(v_{6}\right)\right|=2$. In $S_{5}$, the inclusion of $v_{1}$ does not result in a $\gamma_{s}$-set for the deletion of any element of $S_{5}$. Therefore, $P_{10}$ is not $\gamma_{s}$-very excellent. If $n=7$, then the $\gamma_{s}$-sets are $S_{1}=\left\{v_{2}, v_{5}, v_{7}\right\}, S_{2}=\left\{v_{2}, v_{5}, v_{6}\right\}, S_{3}=\left\{v_{1}, v_{3}, v_{6}\right\}$, 
$S_{4}=\left\{v_{2}, v_{3}, v_{6}\right\}, S_{5}=\left\{v_{2}, v_{4}, v_{6}\right\}$. It can be verified that none of these is a $\gamma_{s}$-very excellent set. Therefore, $P_{7}$ is not a strong very excellent. Therefore, the only $\gamma_{s}$-very excellent paths are $P_{2}$ and $P_{4}$.

Theorem 6. A graph is $\gamma_{s}$-very excellent if and only if there exists a $\gamma_{s}$-set $D$ of $G$ such that to each $u \notin D$ there exist $v \in D$ such that $P N_{w}(v, D) \subseteq N_{w}[u]$.

Proof. Suppose $D$ satisfies this proterty, then clearly $D$ is a very excellent $\gamma_{s}$-set of $G$. Conversely suppose $G$ is $\gamma_{s}$-very excellent. Let $D$ be a very excellent $\gamma_{s}$-set of $G$. Let $u \notin D$. Then there exists a $v \in D$ such that $(D-\{v\}) \cup\{u\}$ is a $\gamma_{s}$-set of $G$. As $(D-\{v\})$ does not strong dominate any vertex of $P N_{w}[v, D]$, and as $(D-\{v\}) \cup\{u\}$ is a $\gamma_{s}$-set, $u$ strong dominates $P N_{w}[v, D]$. That is $P N_{w}[v, D] \subseteq N_{w}[u]$. Hence the theorem.

\section{References}

[1] E. Sampathkumar and Pushpalatha, Strong(Weak) domination and domination balance in graph, Discrete Math. 161, (1996), 235-242.

[2] C. V. R. Harinarayanan et al., Strong domination excellent graphs, submitted.

[3] M. Yamuna, Excellent, just excellent and very excellent graphs, Ph.D, dissertation. Submitted to Alagappa University, Karaikudi, South India, December, 2003.

Department of Mathematics, K.L.N College of Information Technology, Sivagangai, India.

Department of Mathematics, K.L.N College of Engineering, Sivagangai, India.

Department of Mathematics, Mannar Thirumalai Naicker College, Madurai, India.

Department of Mathematics, Rajalakshmi Engineering College, Chennai, India.

Coordinator and Head, Ramanujan Research center, Saraswathi Narayanan College, Madurai, India. 
